\title{
POTENTIALLY DU BOIS SPACES
}

\author{
PATRICK GRAF AND SÁNDOR J KOVÁCS
}

\begin{abstract}
We investigate properties of potentially Du Bois singularities, that is, those that occur on the underlying space of a Du Bois pair. We show that a normal variety $X$ with potentially Du Bois singularities and Cartier canonical divisor $K_{X}$ is necessarily log canonical, and hence Du Bois. As an immediate corollary, we obtain the Lipman-Zariski conjecture for varieties with potentially Du Bois singularities.

We also show that for a normal surface singularity, the notions of Du Bois and potentially Du Bois singularities coincide. In contrast, we give an example showing that in dimension at least three, a normal potentially Du Bois singularity $x \in X$ need not be Du Bois even if one assumes the canonical divisor $K_{X}$ to be $\mathbb{Q}$-Cartier.
\end{abstract}

\section{INTRODUCTION}

Hodge theory of complex projective manifolds has proven to be an extremely useful tool in many different and perhaps a priori unexpected situations. For instance the simple consequence of the degeneration of the Hodge-to-de Rham spectral sequence that the natural map

$$
H^{i}\left(X^{\text {an }}, \mathbb{C}\right) \rightarrow H^{i}\left(X^{\text {an }}, \mathscr{O}_{X^{\text {an }}}\right)
$$

is surjective has many applications. It was discovered early on that this surjectivity continues to hold for normal crossing singularities and even some more complicated singularities. Steenbrink identified the class of singularities that has this property naturally and named them Du Bois singularities Ste83. It turns out that (1.1) along with the requirement that general hyperplane sections of Du Bois singularities should also be Du Bois essentially characterize Du Bois singularities Kov12.

Unfortunately, the rigorous definition of Du Bois singularities is complicated. It relies on a generalization of the de Rham complex, the Deligne-Du Bois complex (see [Kol13, 6.4]), an object in the derived category of coherent sheaves on $X$. However, once the technical difficulties are settled the theory is very powerful.

One possible way to tame Du Bois singularities is to consider this notion a weakening of the notion of rational singularities. In fact, Steenbrink conjectured immediately after introducing the notion that rational singularities are Du Bois and this was confirmed in Kov99.

Originally, Du Bois singularities were introduced to study degenerations of variations of Hodge structures, but Kollár noticed that there is a strong connection between them and the singularities of the minimal model program. In particular, he conjectured that log canonical singularities are Du Bois. This was recently confirmed in KK10. For the definition of the singularities of the minimal model program, such as log canonical and klt, please see [KM98, 2.34] or [Kol13, 2.8].

Date: September 25, 2018.

2010 Mathematics Subject Classification. 14B05, 32S05.

Key words and phrases. Singularities of the minimal model program, Du Bois pairs, differential forms, Lipman-Zariski conjecture.

The first named author was partially supported by the DFG-Forschergruppe 790 "Classification of Algebraic Surfaces and Compact Complex Manifolds".

The second named author was supported in part by NSF Grant DMS-1301888, a Simons Fellowship (\#304043), and the Craig McKibben and Sarah Merner Endowed Professorship in Mathematics at the University of Washington. This work was partially completed while he enjoyed the hospitality of the Institute for Advanced Study (Princeton) supported by The Wolfensohn Fund. 
The evolution of the minimal model program taught us that singularities should be studied in pairs, that is, instead of considering a single space $X$ one should consider a pair consisting of a variety and a subvariety. This has also proved to be a powerful generalization.

The notion of Du Bois singularities was recently generalized for pairs $(X, \Sigma)$ consisting of a complex variety $X$ and a closed subscheme $\Sigma \subset X$ Kov11. For a relatively detailed treatment the reader should peruse [Kol13, Chapter 6].

Recently Du Bois singularities and Du Bois pairs have provided useful tools in many situations. For instance, they form a natural class of singularities where Kodaira type vanishing theorems hold Ste85, KSS10, Kov11, Kov13b, Pat13. Other recent applications include extension theorems GKKP11, positivity theorems Sch12], categorical resolutions Lun12, log canonical compactifications [HX13, semipositivity [FFS13, and injectivity theorems [Fuj13]. Besides applications in the minimal model program, $\mathrm{Du}$ Bois singularities play an important role in moduli theory as well [KK10, Kov13b].

The introduction of $\mathrm{Du}$ Bois pairs was motivated by the success of studying singularities of pairs in the minimal model program. If a pair $(X, \Delta)$ is log canonical (resp. klt) and $\Delta$ is $\mathbb{Q}$-Cartier, then $X$ is also log canonical (resp. klt). An analogous statement is not straightforward for Du Bois pairs. The fact that $(X, \Sigma)$ is a Du Bois pair does not clearly imply that then $X$ itself is Du Bois. In fact, one of the advantages of $\mathrm{Du}$ Bois pairs is that they provide a possibility to extend the power of Hodge theoretic techniques to a larger class of varieties. In other words, it is natural to ask and potentially helpful to know the answer to the following question.

Question 1.2. Given a complex variety $X$, when does there exist a subvariety $\Sigma \subsetneq X$ such that $(X, \Sigma)$ is a Du Bois pair?

To make it easier to discuss these singularities, we introduce the following definition:

Definition 1.3. Let $X$ be a complex variety and $x \in X$ a closed point. We say that $X$ is potentially $D u$ Bois at $x$ if there exists a Zariski-open set $U \subseteq X$ containing $x$ and a subvariety $\Sigma_{U} \subseteq U$ not containing any irreducible components of $U$ such that $\left(U, \Sigma_{U}\right)$ is a Du Bois pair (see Section $1 . \mathrm{D}$ for the definition of Du Bois pairs). $X$ is called potentially Du Bois if it is potentially Du Bois at $x$ for every closed point $x \in X$.

The main result of this paper gives some answers to Question 1.2 .

Theorem 1.4. Let $X$ be a normal complex variety. Then:

(1.4.1) If $X$ has potentially Du Bois singularities, then it is Du Bois in codimension two, i.e. the non-Du Bois locus of $X$ has codimension at least three.

(1.4.2) Let $(X, \Sigma)$ be a Du Bois pair and $\Delta \subset X$ a reduced effective divisor such that $\operatorname{supp} \Delta \subseteq \Sigma$ and $K_{X}+\Delta$ is Cartier. Then $(X, \Delta)$ is log canonical and hence $X$ is Du Bois. In particular, if $X$ has potentially Du Bois singularities and $K_{X}$ is Cartier, then it is log canonical and Du Bois.

(1.4.3) There exists a three-dimensional normal variety $X$ with isolated singularities and $\mathbb{Q}$-Cartier canonical divisor such that $X$ has potentially $D u$ Bois singularities, but it is not Du Bois. In particular, the bound on the codimension in (1.41) is sharp.

We have the following immediate corollary of Theorem 1.412 ,

Corollary 1.5 (Lipman-Zariski conjecture for potentially Du Bois singularities). Let X be a complex variety with potentially Du Bois singularities. If the tangent sheaf $\mathscr{T}_{X}:=\mathscr{H}_{\mathrm{om}} \mathscr{O}_{X}\left(\Omega_{X}^{1}, \mathscr{O}_{X}\right)$ is locally free, then $X$ is smooth.

For normal singularities, Theorem 1.41] and 1.42 give a complete answer to Question 1.2 if either $K_{X}$ is Cartier or the dimension of $X$ is two. Theorem 1.43 can also be interpreted as a positive result: it says that in higher dimensions, we stand a chance of finding a suitable $\Sigma \subset X$ making $(X, \Sigma)$ into a Du Bois pair, even if $K_{X}$ is $\mathbb{Q}$-Cartier.

Let us now put Theorem 1.4 in a broader philosophical perspective. It is well known that for singularities in the minimal model program, decreasing the boundary divisor by a $\mathbb{Q}$-Cartier divisor improves the singularities of the pair. In particular, as mentioned above, if $K_{X}$ is $\mathbb{Q}$-Cartier, then $(X, \Delta)$ being klt, dlt, lc, etc. implies that $X$ satisfies the same condition. Theorem 1.43 tells us that this principle does 
not hold for Du Bois singularities. It is relatively easy to give an example of a non-normal singularity which is potentially Du Bois, but not Du Bois [KS13, Ex. 2.10]. It is a little more complicated to give a normal example of the same behavior [KS13, Ex. 2.14]. Then it is much harder to give an example with a $\mathbb{Q}$-Cartier canonical divisor. Our example in 1.413 does exactly that.

Another distinction between singularities of the minimal model program and Du Bois singularities is that the former depend on the behavior of $m K_{X}$, a multiple of the canonical divisor for a sufficiently divisible $m \in \mathbb{N}$, while rational and Du Bois singularities depend on the behavior of the canonical divisor itself. This distinction manifests itself in the fact that rational singularities whose canonical divisor is Cartier are canonical, but a rational singularity with only a $\mathbb{Q}$-Cartier canonical divisor does not need to be even log canonical. Theorem 1.4/2 and 1.413 demonstrate that this phenomenon also happens for Du Bois singularities.

Remark 1.6. Our example in (1.43) has a $\mathbb{Q}$-Cartier canonical divisor of index 4. One might ask whether there also exist examples of index 2 or 3 . We conjecture that this is the case, however it is not clear whether our construction can be adapted to yield such examples.

1.A. Outline of proofs. For Theorem [1.41], in the surface case we prove the statement directly and then we conclude the general case using the deformation invariance of Du Bois singularities KS12. For Theorem 1.42, we apply the vanishing theorem of [Kov13b, 5.3] together with the techniques from [GKKP11, Section 17] in order to obtain an extension theorem for reflexive differentials, Theorem 4.1] from which the claim immediately follows.

Theorem 1.433 has the most involved proof and actually the bulk of this paper is devoted to the construction of the example whose existence is claimed there. The basic idea is to take a cone over a smooth projective surface $T$. So we first need a criterion for a cone (or a pair of cones) to be Du Bois. It turns out that such a criterion can be phrased in terms of the cohomology of certain line bundles on $T$. It is well-known that a cone over $T$ can have a $\mathbb{Q}$-Cartier canonical divisor only if $K_{T}$ is either anti-ample, torsion, or ample. Furthermore, in the first two cases, the cone is automatically Du Bois, even $\log$ canonical. Hence our example $T$ must necessarily be canonically polarized.

In order to construct $T$, first we find a ruled surface $S$ having the required cohomological properties. Then $T$ is defined to be the general member of a suitable linear system in the product $S \times B$, where $B$ is a curve of genus 2. The tricky part is to show that $T$ is smooth although it lives in a linear system with a non-empty base locus.

1.B. Acknowledgements. We would like to thank Clemens Jörder, Stefan Kebekus, and Karl Schwede for interesting discussions on the subject of this paper. Furthermore we would like to thank the anonymous referee for providing suggestions that made the paper more accessible.

1.C. Notation, definitions, and conventions. Throughout this paper, we work over the field of complex numbers $\mathbb{C}$.

A reduced pair $(X, \Sigma)$ consists of $X$ a reduced scheme of finite type over $\mathbb{C}$ and a reduced closed subscheme $\Sigma \subset X$. Neither $X$ nor $\Sigma$ is assumed to be pure dimensional or normal.

Let $(X, \Sigma)$ be a reduced pair and $x \in X$ a point. We say that $(X, \Sigma)$ is snc at $x$ if there exists a Zariski-open neighbourhood $U \subseteq X$ of $x$ such that $U$ is smooth and $\Sigma \cap U$ is either empty, or a divisor with simple normal crossings. The pair $(X, \Sigma)$ is called an snc pair or simply snc if it is snc at every point of $X$.

Given a reduced pair $(X, \Sigma),(X, \Sigma)_{\text {reg }}$ will denote the maximal open subset of $X$ where $(X, \Sigma)$ is snc, and $(X, \Sigma)_{\text {sing }}$ its complement, with the induced reduced subscheme structure.

A reduced pair $(X, \Sigma)$ is called normal if $X$ is normal and projective if $X$ is projective.

If $(X, \Sigma)$ is a normal reduced pair, then by definition $X$ is smooth in codimension 1 . Furthermore, since $\Sigma$ is reduced, near a general point of $\Sigma$, both $X$ and $D$ are smooth. In particular in this case, $\operatorname{codim}_{X}(X, \Sigma)_{\text {sing }} \geq 2$, or in other words $(X, \Sigma)$ is snc in codimension 1 .

Let $(X, \Sigma)$ be a normal reduced pair. A log resolution of $(X, \Sigma)$ is a proper birational morphism $\pi: \widetilde{X} \rightarrow X$ such that $\widetilde{X}$ is smooth, both the pre-image $\pi^{-1}(\Sigma)$ of $\Sigma$ and the exceptional set $E=\operatorname{Exc}(\pi)$ are of pure codimension 1 in $\widetilde{X}$, and $(\widetilde{X}, \widetilde{D}+E)$ is an snc pair where $\widetilde{D}=\pi^{-1}(\Sigma)_{\text {red }}$ is the reduced divisor supported on $\pi^{-1}(\Sigma)$. 
Let $D, D_{1}, D_{2}$ be divisors on a normal variety. Then $D_{1} \vee D_{2}$ denotes the smallest divisor that contains both $D_{1}$ and $D_{2}$ and $D_{1} \wedge D_{2}$ denotes the largest divisor that is contained in both $D_{1}$ and $D_{2}$. Finally, we will use the shorthand $h^{i}(X, D)$ to denote $\operatorname{dim}_{\mathbb{C}} H^{i}\left(X, \mathscr{O}_{X}(D)\right)$.

1.D. Du Bois singularities and Du Bois pairs. The Deligne-Du Bois complex DB81] associated to a complex variety $X$ is a filtered complex $\underline{\Omega}_{X}^{*}$, unique up to quasi-isomorphism, which for a smooth $X$ is isomorphic to the de Rham complex considered with the stupid filtration. Many of the usual cohomological properties of the de Rham complex that hold for smooth varieties remain true for arbitrary varieties if one replaces the de Rham complex with the Deligne-Du Bois complex. In particular, if $X$ is proper, then there is a Frölicher-type spectral sequence converging to singular cohomology and degenerating at $E_{1}$. We say that $X$ has Du Bois singularities if the zeroth graded piece of $\underline{\Omega}_{X}^{\circ}$ is quasiisomorphic to $\mathscr{O}_{X}$. It follows that if $X$ is proper and has Du Bois singularities, then the natural map $H^{i}\left(X^{\text {an }}, \mathbb{C}\right) \rightarrow H^{i}\left(X^{\text {an }}, \mathscr{O}_{X^{\text {an }}}\right)$ is surjective. In fact, this property is close to characterizing Du Bois singularities, cf. Kov12. Note however that Du Bois varieties in general are not necessarily normal and hence may have singularities in codimension 1. For example normal crossing singularities are Du Bois.

We briefly explain the construction of the (zeroth graded piece of the) Deligne-Du Bois complex. Given a singular variety $X$, first we resolve its singularities by a log resolution $\pi$. However this is not enough, we also need to resolve the singularities of the singular locus of $X$ and those of the exceptional set of $\pi$, and then we need to resolve the exceptional sets of these resolutions, and so on. To do this properly one ends up with a diagram of morphisms in the shape of a $(\operatorname{dim} X+1)$-dimensional hypercube, or more precisely a "cubical hyperresolution" of $X$ cf. Car85, GNPP88, PS08. Similarly to the way we associate a simple complex to a double complex, taking disjoint unions of certain objects in this diagram yields a "semi-simplicial variety $X$. with a morphism $\varepsilon_{\bullet}: X_{\bullet} \rightarrow X$ ". On every component of $X$., we may consider an injective resolution of the structure sheaf, and we may put all these resolutions together into a double complex using the pull-back and push-forward maps between the components of $X$. . Now by applying $\varepsilon_{*}$ to this double complex and forming the associated simple complex, we obtain the derived push-forward $R \varepsilon_{*} \mathscr{O}_{X}$. This is exactly the zeroth graded piece of the Deligne-Du Bois complex. For a more detailed, yet still down-to-earth introduction see [Ste85, §2], for a rigorous treatment see [DB81, GNPP88, or the more recent PS08, §7.3].

Du Bois pairs were introduced in Kov11. Their definition is as involved as the definition of Du Bois singularities, so we will not repeat it here. The essential ingredient is the following: For any reduced pair $(X, \Sigma)$ there exists an object in the filtered derived category of $X$, called the Deligne-Du Bois complex of $(X, \Sigma)$ that relates the Deligne-Du Bois complex of $X$ and that of $\Sigma$. If $(X, \Sigma)$ is an snc pair, then the Deligne-Du Bois complex of $(X, \Sigma)$ is quasi-isomorphic to $\Omega_{X}^{\bullet}(\log \Sigma)(-\Sigma)$. A reduced pair $(X, \Sigma)$ is a Du Bois pair if the associated zeroth graded quotient of the Deligne-Du Bois complex of $(X, \Sigma)$ is quasi-isomorphic to the ideal sheaf of $\Sigma$ in $\mathscr{O}_{X}$. For more on Du Bois pairs see [Kov11] and [Kol13, $\S 6$ ].

As we already defined in Definition 1.3, a variety $X$ is said to have potentially Du Bois singularities if there exists a cover of $X$ by Zariski-open subsets $U_{i} \subset X$ such that for any $i$, there exists a subvariety $\Sigma_{i} \subset U_{i}$ not containing any irreducible component of $U_{i}$ and making $\left(U_{i}, \Sigma_{i}\right)$ into a Du Bois pair.

\section{Two Du Bois CRITERIA}

In this section, we give two necessary and sufficient criteria for pairs of a certain kind to be Du Bois. The first criterion is concerned with varieties with only isolated non-Du Bois points, while the second one, a corollary of the first, deals with cones over Du Bois pairs $(X, \Sigma)$. The latter criterion is likely known to experts in some form at least in the case $\Sigma=\emptyset$. In that case a similar statement was proved in Ma13, Thm. 4.4].

Theorem 2.1 (A Du Bois criterion for isolated non-Du Bois locus). Let (X, $\Sigma$ ) be a normal reduced pair with a closed point $x \in X$ such that $(X \backslash\{x\}, \Sigma \backslash\{x\})$ is a Du Bois pair. Let $f: Y \rightarrow X$ be a proper birational morphism from a normal variety $Y$ that is an isomorphism over $X \backslash\{x\}$. Let $E$ denote the (not necessarily divisorial) exceptional locus of $f$, and set $\Gamma=E \cup f^{-1}(\Sigma)$. Assume that $(Y, \Gamma)$ is a Du Bois pair. Then $(X, \Sigma)$ is a Du Bois pair if and only if

$$
R^{i} f_{*} \mathscr{O}_{Y}(-\Gamma)=0
$$


for all $i \geq 1$.

2.A. Cones over pairs. First we recall some basic facts about cones, and we fix the notation used in Theorem 2.5. We will follow the conventions and notation of [Kol13, §3.1].

Notation 2.2 (Affine cones). Let $X$ be a projective scheme and $\mathscr{L} \in \operatorname{Pic} X$ an ample line bundle on $X$. The affine cone over $X$ with respect to $\mathscr{L}$ is

$$
C_{a}(X, \mathscr{L})=\operatorname{Spec} R(X, \mathscr{L})
$$

where

$$
R(X, \mathscr{L})=\bigoplus_{n \geq 0} H^{0}\left(X, \mathscr{L}^{n}\right)
$$

is the section ring of $\mathscr{L}$. If there is no ambiguity about the choice of $\mathscr{L}$, we will write $C X$ for $C_{a}(X, \mathscr{L})$. If $X$ is connected, the vertex $P \in C X$ is defined to be the closed point corresponding to the maximal ideal

$$
\bigoplus_{n \geq 1} H^{0}\left(X, \mathscr{L}^{n}\right) \subset R(X, \mathscr{L})
$$

Remark 2.3. See [Kol13, §3.1] for generalities on cones. Note that this construction works even if $\mathscr{L}$ is not very ample. If $X$ is normal and $\mathscr{L}$ is very ample, then $C_{a}(X, \mathscr{L})$ is the normalization of the classical affine cone over the embedding of $X$ via $\mathscr{L}$. In particular, $C_{a}(X, \mathscr{L})$ is isomorphic to the classical affine cone if and only if the embedding given by $\mathscr{L}$ is projectively normal. Notice further that $C_{a}(X, \mathscr{L})$ is normal whenever $X$ is normal, even if $\mathscr{L}$ is not very ample.

Notation 2.4 (Pairs of cones). Let $X$ be a normal projective variety, $\Sigma \subset X$ a reduced subscheme, and $\mathscr{L} \in \operatorname{Pic} X$ an ample line bundle on $X$. There is a natural map $\iota: C_{a}\left(\Sigma,\left.\mathscr{L}\right|_{\Sigma}\right) \rightarrow C_{a}(X, \mathscr{L})$, which is a closed embedding away from the vertex $P \in C X$. We will also denote $C_{a}(X, \mathscr{L})$ by $C X$, the image of $\iota$ by $C \Sigma$, and the pair of cones consisting of $C X$ and $C \Sigma$ by $C_{a}(X, \Sigma, \mathscr{L})$, or for simplicity by $(C X, C \Sigma)$.

Theorem 2.5 (A Du Bois criterion for cones). Let $(X, \Sigma)$ be a normal projective Du Bois pair and $\mathscr{L}$ an ample line bundle on $X$. Then the pair of cones $C_{a}(X, \Sigma, \mathscr{L})$ is a Du Bois pair if and only if

$$
H^{i}\left(X, \mathscr{L}^{n}(-\Sigma)\right)=0
$$

for all $i, n \geq 1$.

2.B. Proof of Theorem 2.1. First assume that $\Sigma=\emptyset$. In this case, $\Gamma=E$. Consider the following commutative diagram of distinguished triangles:

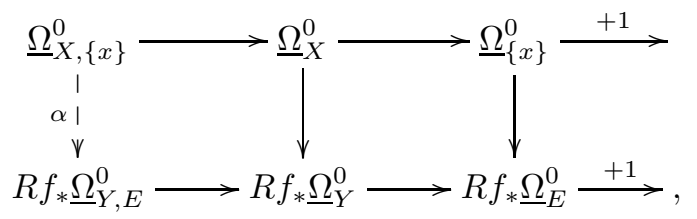

where the solid arrows are the obvious natural maps and $\alpha$ is the induced map that keeps the diagram commutative cf. Kov11, Prop. 3.11]. Next consider the distinguished triangle from [DB81, Prop. 4.11]:

$$
\underline{\Omega}_{X}^{0} \longrightarrow \underline{\Omega}_{\{x\}}^{0} \oplus R f_{*} \underline{\Omega}_{Y}^{0} \longrightarrow R f_{*} \underline{\Omega}_{E}^{0} \stackrel{+1}{\longrightarrow},
$$

and observe that combined with [KK10, (2.1.4)] this implies that $\alpha$ is an isomorphism and hence there exists the following distinguished triangle:

$$
R f_{*} \underline{\Omega}_{Y, E}^{0} \longrightarrow \underline{\Omega}_{X}^{0} \longrightarrow \underline{\Omega}_{\{x\}}^{0} \stackrel{+1}{\longrightarrow}
$$

Since $(Y, E)$ is a Du Bois pair by assumption, $\underline{\Omega}_{Y, E}^{0} \simeq \mathscr{O}_{Y}(-E)$ and since $X$ is normal (for this seminormal would be enough), it follows by [Sai00, Prop. 5.2] (cf. [Sch09, 5.6], [KS11, 7.6]) that $h^{0}\left(\underline{\Omega_{X}^{0}}\right) \simeq$ 
$\mathscr{O}_{X}$ and hence we have the following long exact sequence:

$$
0 \rightarrow f_{*} \mathscr{O}_{Y}(-E) \rightarrow \mathscr{O}_{X} \rightarrow \mathscr{O}_{\{x\}} \rightarrow R^{1} f_{*} \mathscr{O}_{Y}(-E) \rightarrow h^{1}\left(\underline{\Omega}_{X}^{0}\right) \rightarrow \underbrace{h^{1}\left(\underline{\Omega}_{\{x\}}^{0}\right)}_{=0} \rightarrow \ldots
$$

Since $\mathscr{O}_{X} \rightarrow \mathscr{O}_{\{x\}}$ is surjective, we conclude that $R^{i} f_{*} \mathscr{O}_{Y}(-E) \simeq h^{i}\left(\underline{\Omega}_{X}^{0}\right)$ for $i \geq 1$ and hence in this case $X$ (or equivalently $(X, \Sigma)$ ) is Du Bois if and only if

$$
R^{i} f_{*} \mathscr{O}_{Y}(-E)=0 \quad \text { for } i \geq 1
$$

We will now turn to the case $\Sigma \neq \emptyset$. In particular, since the statement is local, we may assume that $x \in \Sigma$. Again by [DB81, Prop. 4.11], we have a diagram of distinguished triangles:

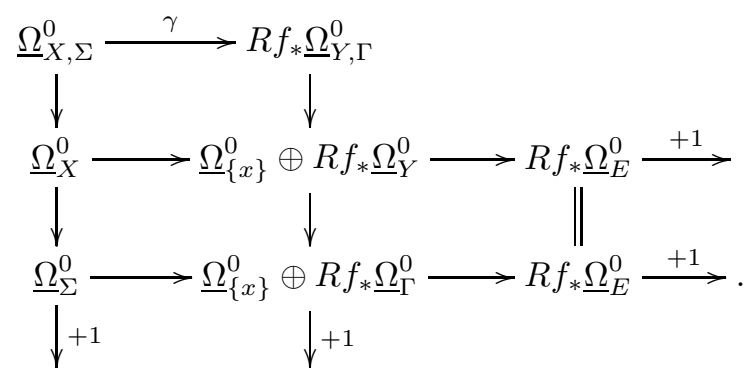

It follows from [Kov13a, (B.1.1)] that $\gamma$ is an isomorphism. Since we assumed $(Y, \Gamma)$ to be a Du Bois pair, we obtain an isomorphism

$$
\underline{\Omega}_{X, \Sigma}^{0} \simeq R f_{*} \mathscr{O}_{Y}(-\Gamma) .
$$

So $(X, \Sigma)$ is a Du Bois pair if and only if

$$
f_{*} \mathscr{O}_{Y}(-\Gamma) \simeq \mathscr{I}_{\Sigma \subset X} \text { and } R^{i} f_{*} \mathscr{O}_{Y}(-\Gamma)=0 \text { for } i \geq 1 .
$$

Observe that there exists a short exact sequence:

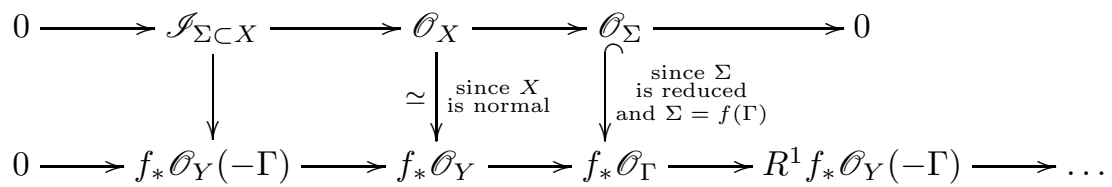

It follows that the image of $f_{*} \mathscr{O}_{Y}$ in $f_{*} \mathscr{O}_{\Gamma}$ is exactly $\mathscr{O}_{\Sigma}$ and hence $f_{*} \mathscr{O}_{Y}(-\Gamma) \simeq \mathscr{I}_{\Sigma \subset X}$ always holds. So we obtain that $(X, \Sigma)$ is a Du Bois pair if and only if

$$
R^{i} f_{*} \mathscr{O}_{Y}(-\Gamma)=0 \text { for } i \geq 1 .
$$

Notice that if $\Sigma=\emptyset$, then $\Gamma=E$ so the conditions (2.7) and (2.8) are actually the same whether $\Sigma=\emptyset$ or $\Sigma \neq \emptyset$. This proves Theorem 2.1.

2.C. Proof of Theorem 2.5. Let $f: Y=\operatorname{Spec}_{X} \bigoplus_{m>0} \mathscr{L}^{m} \rightarrow C X$ be a weighted blowup of the vertex $P \in C X$, with exceptional divisor $E \subset Y$ (cf. [Kol13, p.98]). Then $Y$ is the total space of the dual bundle $\mathscr{L}^{-1}$ and hence the natural map $\pi: Y \rightarrow X$ is a smooth affine morphism. Let $Z:=\pi^{-1}(\Sigma)$ and $\Gamma:=Z \cup E$.

Lemma 2.9. $(Y, \Gamma)$ is a Du Bois pair.

Proof. Since $(X, \Sigma)$ is a Du Bois pair, it follows from [Kol13, 6.19] that $(Y, Z)$ is a Du Bois pair and from Kol13, 6.17] that $(\Gamma, Z)$ is a Du Bois pair. Hence, the second and third rows of the following diagram 
form distinguished triangles.

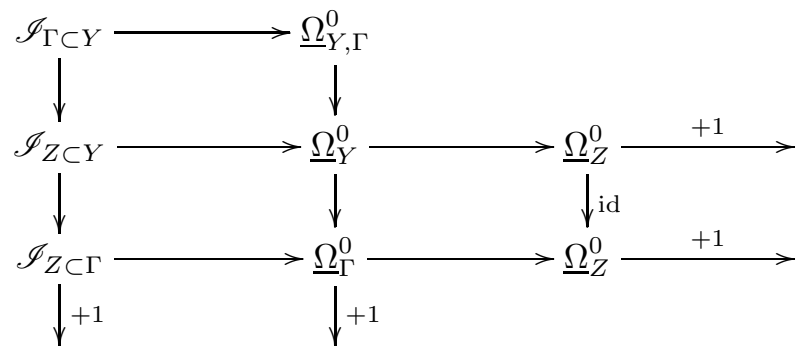

The first column is an obvious short exact sequence of ideals and the second is a distinguished triangle by definition. Therefore, [Kov13a, (B.1.1)] implies that $\underline{\Omega}_{Y, \Gamma}^{0} \simeq \mathscr{I}_{\Gamma \subset Y}$, so indeed $(Y, \Gamma)$ is Du Bois.

Proof of Theorem 2.5 continued. By Lemma 2.9 and Theorem 2.1. $(C X, C \Sigma)$ is a Du Bois pair if and only if $R^{i} f_{*} \mathscr{O}_{Y}(-\Gamma)=0$ for all $i \geq 1$, so we need to prove that this vanishing is equivalent to (2.6).

First notice that $R^{i} f_{*} \mathscr{O}_{Y}(-\Gamma)$ is a skyscraper sheaf supported on $P \in C X$, with stalk

$$
H^{i}\left(Y, \mathscr{O}_{Y}(-\Gamma)\right) \simeq H^{i}\left(X, \pi_{*} \mathscr{O}_{Y}(-\Gamma)\right),
$$

where the isomorphism follows because $\pi$ is an affine morphism. In the remainder of the proof we will demonstrate that

$$
\pi_{*} \mathscr{O}_{Y}(-\Gamma) \simeq \bigoplus_{n \geq 1} \mathscr{L}^{n}(-\Sigma)
$$

which, combined with (2.10), implies the desired statement.

Recall that $\Gamma=Z \cup E$ and $\pi$ is affine and consider the following diagram of short exact sequences:

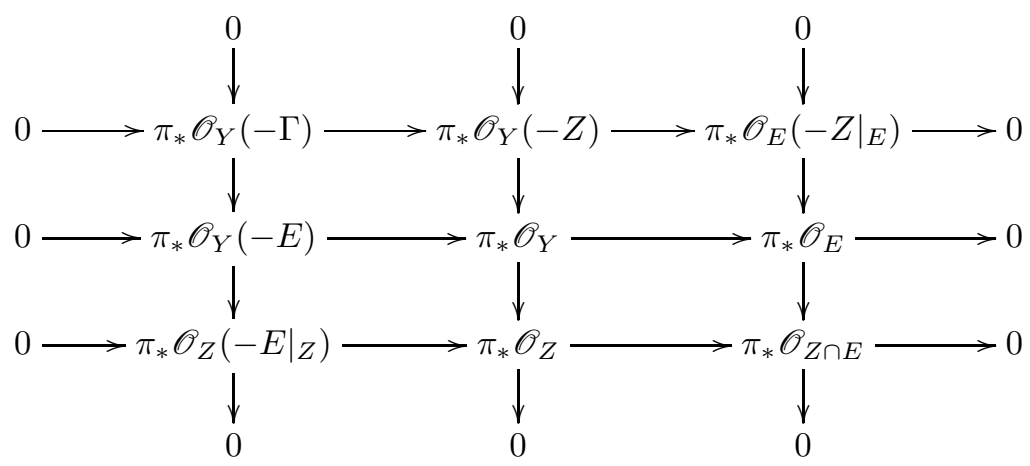

By construction

SO

$$
\pi_{*} \mathscr{O}_{Y} \simeq \bigoplus_{n \geq 0} \mathscr{L}^{n}, \quad \text { and }\left.\quad \pi_{*} \mathscr{O}_{Z} \simeq \bigoplus_{n \geq 0} \mathscr{L}^{n}\right|_{\Sigma}
$$

$$
\pi_{*} \mathscr{O}_{Y}(-Z) \simeq \bigoplus_{n \geq 0} \mathscr{L}^{n}(-\Sigma) .
$$

It is easy to see that $\pi$ induces isomorphisms $E \simeq X$ and $Z \cap E \simeq \Sigma$, and hence

$$
\pi_{*} \mathscr{O}_{E} \simeq \mathscr{O}_{X} \quad \text { and } \quad \pi_{*} \mathscr{O}_{Z \cap E} \simeq \mathscr{O}_{\Sigma},
$$

which implies that

$$
\pi_{*} \mathscr{O}_{E}\left(-\left.Z\right|_{E}\right) \simeq \mathscr{O}_{X}(-\Sigma) \simeq \mathscr{L}^{0}(-\Sigma) .
$$

Finally (2.12), (2.13), and (2.14) together imply (2.11) and hence Theorem 2.5 follows. 


\section{Proof of Theorem (1.4, 1)}

Proposition 3.1. Let $(X, \Sigma)$ be a Du Bois pair, where $X$ is a normal surface. Then $X$ is Du Bois.

Proof. We may assume that $X$ has a single isolated singularity $x \in X$, that $\Sigma$ is a divisor and that $x \in \Sigma$. Let $\pi: Y \rightarrow X$ be a $\log$ resolution of $(X, \Sigma)$, with exceptional set $E \subset Y$, and let $\Gamma \subset Y$ be the preimage of $\Sigma$. Notice that $\Gamma=E+T$, where $T:=\pi_{*}^{-1} \Sigma$ is the strict transform of $\Sigma$.

By Theorem 2.1 applied to $(X, \emptyset)$, we have that $X$ is Du Bois if and only if

$$
R^{1} \pi_{*} \mathscr{O}_{Y}(-E)=0,
$$

while $(X, \Sigma)$ is a Du Bois pair if and only if

$$
R^{1} \pi_{*} \mathscr{O}_{Y}(-\Gamma)=0 .
$$

So it suffices to show that (3.1.2) implies (3.1.1). To this end, consider the short exact sequence

$$
0 \rightarrow \mathscr{O}_{Y}(-\Gamma) \rightarrow \mathscr{O}_{Y}(-E) \rightarrow \mathscr{O}_{T}(-E) \rightarrow 0
$$

and apply $\pi_{*}$. The associated long exact sequence gives the following:

$$
\cdots \rightarrow R^{1} \pi_{*} \mathscr{O}_{Y}(-\Gamma) \rightarrow R^{1} \pi_{*} \mathscr{O}_{Y}(-E) \rightarrow R^{1} \pi_{*} \mathscr{O}_{T}(-E) \rightarrow \ldots
$$

However, $R^{1} \pi_{*} \mathscr{O}_{T}(-E)=0$ because $\left.\pi\right|_{T}$ is a finite morphism, and then (3.1.2) implies the desired statement.

Proof of Theorem (1.41). After shrinking $X$, we may assume that $X$ is affine and that there is a subvariety $\Sigma \subset X$ such that $(X, \Sigma)$ is a Du Bois pair. Let $H \subset X$ be the intersection of $n-2$ general hyperplanes in $X$, where $n$ is the dimension of $X$. Then $\left(H,\left.\Sigma\right|_{H}\right)$ is a Du Bois pair by repeated application of Lemma 4.4. By Proposition 3.1 it follows that $H$ is Du Bois. A repeated application of KS12, Thm. 4.1] now shows that $X$ is Du Bois near $H$. So the non-Du Bois locus of $X$ does not intersect a general complete intersection surface in $X$. It follows that the non-Du Bois locus has codimension at least three.

\section{Proof of Theorem (1.4,2)}

Theorem (1.42) is an immediate consequence of the following theorem, which for log canonical pairs was proved in [GKKP11, Theorem 16.1].

Theorem 4.1 (Extension theorem for $p$-forms on Du Bois pairs). Let $(X, \Sigma)$ be a Du Bois pair, where $X$ is normal. If $\pi: \widetilde{X} \rightarrow X$ is a log resolution of $(X, \Sigma)$ with exceptional divisor $E=\operatorname{Exc}(\pi)$, then the sheaves

$$
\pi_{*} \Omega_{\widetilde{X}}^{p}(\log \widehat{D}), \quad 0 \leq p \leq n,
$$

are reflexive, where $\widehat{D}=\left(\pi^{-1}(\Sigma) \vee E\right)_{\mathrm{red}}$, the reduced divisor with support $\pi^{-1}(\Sigma) \cup \operatorname{supp} E \subset \widetilde{X}$.

Proof of Theorem (1.42). We will use the notation from Theorem 4.1 above. Write $\Sigma=\Sigma_{\text {div }} \cup \Sigma_{\text {non-div }}$ as the union of closed sets such that $\Sigma_{\text {div }}$ is a divisor and $\operatorname{codim}_{X} \Sigma_{\text {non-div }} \geq 2$. Let $Z=\pi(E) \cup \Sigma_{\text {non-div }}$ and $U=X \backslash Z$. It follows that $\left.\left.\left(\pi_{*} \omega_{\widetilde{X}}(\widehat{D})\right)\right|_{U} \simeq\left(\omega_{X}\left(\Sigma_{\text {div }}\right)\right)\right|_{U}$, and applying Theorem 4.1 in the case $p=\operatorname{dim} X$ we obtain that $\pi_{*} \omega_{\tilde{X}}(\widehat{D})$ is reflexive. Since $X$ is normal, $\operatorname{codim}_{X} Z \geq 2$, and hence $\pi_{*} \omega_{\widetilde{X}}(\widehat{D}) \simeq \omega_{X}\left(\Sigma_{\text {div }}\right)$.

Now, by assumption supp $\Delta \subset \Sigma$ and $K_{X}+\Delta$ is Cartier. Therefore $\omega_{X}(\Delta) \subseteq \omega_{X}\left(\Sigma_{\text {div }}\right) \simeq \pi_{*} \omega_{\widetilde{X}}(\widehat{D})$. In other words there exists a non-zero morphism $\omega_{X}(\Delta) \rightarrow \pi_{*} \omega_{\tilde{X}}(\widehat{D})$ which is an isomorphism on a non-empty open subset of $X$. By adjointness this implies the existence of a non-zero morphism $\pi^{*} \omega_{X}(\Delta) \rightarrow \omega_{\widetilde{X}}(\widehat{D})$ which is an isomorphism on a non-empty open subset of $\widetilde{X}$. Since $\omega_{X}(\Delta)$ is a line bundle, this means that $\pi^{*} \omega_{X}(\Delta) \rightarrow \omega_{\widetilde{X}}(\widehat{D})$ is actually injective, which means that all the discrepancies of the pair $(X, \Delta)$ are at least -1 , that is, $(X, \Delta)$ is $\log$ canonical. 
4.A. Steenbrink-type vanishing results. Next we turn to the proof of Theorem 4.1 In fact, the argument used to prove GKKP11, Thm. 16.1] works in this case essentially unchanged, provided the ingredients of that proof are adapted to the present situation.

The proof of Theorem 4.1 relies on Steenbrink-type vanishing results which, again, were already proved in [GKKP11, Sec. 14] for log canonical pairs. Here we need the following more general statement:

Theorem 4.2 (Kov13b, 5.3], cf. GKKP11, Theorem 14.1]). Let $(X, \Sigma)$ be a Du Bois pair and assume that $\operatorname{dim} X \geq 2$. Further let $\pi: \widetilde{X} \rightarrow X$ be a log resolution of $(X, \Sigma)$ with exceptional divisor $E=\operatorname{Exc}(\pi)$ and let $\widehat{D}=\left(\pi^{-1}(\Sigma) \vee E\right)_{\text {red. }}$ Then we have

$$
R^{n-1} \pi_{*}\left(\Omega_{\widetilde{X}}^{p}(\log \widehat{D}) \otimes \mathscr{O}_{\widetilde{X}}(-\widehat{D})\right)=0
$$

for all $0 \leq p \leq n$.

Corollary 4.3 (cf. GKKP11, Corollary 14.2]). Let $(X, \Sigma)$ be a Du Bois pair and assume that $\operatorname{dim} X \geq 2$. Further let $\pi: \widetilde{X} \rightarrow X$ be a log resolution of $(X, \Sigma)$ with exceptional divisor $E=\operatorname{Exc}(\pi), \widehat{D}=\left(\pi^{-1}(\Sigma) \vee\right.$ $E)_{\mathrm{red}}$, and $x \in X$ a point with reduced fibre $F_{x}=\pi^{-1}(x)_{\mathrm{red}}$. Then

$$
H_{F_{x}}^{1}\left(\widetilde{X}, \Omega_{\widetilde{X}}^{p}(\log \widehat{D})\right)=0 \quad \text { for all } 0 \leq p \leq n .
$$

Proof. This follows from Theorem 4.2 by applying duality for cohomology with support [GKK10, Theorem A.1].

4.B. Proof of Theorem 4.1, We will need the following technical lemma.

Lemma 4.4 (Bertini theorem for Du Bois pairs). Let $(X, \Sigma)$ be a Du Bois pair, $H \in|L|$ a general member of a basepoint-free linear system, and $\Sigma_{H}:=\operatorname{supp}(\Sigma \cap H)$. Then $\left(H, \Sigma_{H}\right)$ is also a Du Bois pair.

Proof. This is proved in [Kov11, 3.18] (cf. [Kol13, 6.5.6]).

Proof of Theorem [4.1. We mainly follow the proof given in [GKKP11, Section 17] with some adjustements. Here we explain the main ideas of that proof with the necessary changes. The reader is referred to GKKP11] for technical details.

The proof works by proving the extension statement one-by-one over the irreducible components $E_{0}$ of the exceptional locus $E$ of a $\log$ resolution $\pi: \widetilde{X} \rightarrow X$ of $X$. The argument follows a double induction on the dimension of $X$ and the codimension of the image $\pi\left(E_{0}\right) \subset X$. There are two main techniques used in the proof. The first one is extending sections from an open set to an ambient set by using vanishing of the local cohomology group that connects the two. This is exactly what is provided by Theorem 4.2 and Corollary 4.3 which replace [GKKP11, Corollary 14.2] in the original proof. The other main tool is cutting by hyperplane sections and in order for that to be effective we need a Bertini type statement. This is provided by Lemma 4.4,

Following the proof in GKKP11, Section 17] the first issue we need to deal with is that here $\Sigma$ is not assumed to be a divisor. This is however not a real problem. In the original proof $\Sigma$ only needs to be a divisor so the notion of being $\log$ canonical make sense. It is never used that $\Sigma$ is a divisor in any other way, so the arguments still make sense if one replaces the words "log canonical" with "Du Bois pair".

For the start of the induction, that is $\operatorname{dim} X=2$, we use Corollary 4.3. As mentioned above, the point of this step as well as the heart of the entire proof is that we are able to extend sections from an open set to an ambient set if we have a vanishing of the local cohomology group that connects the two.

Similarly, the setup and the simplifications performed in the inductive step work fine until we need to use the vanishing of the appropriate local cohomology groups. There we need to substitute Corollary 4.3 for [GKKP11, Corollary 14.2] in the original argument. The same needs to be done with all further occurrences of that corollary.

We also need to replace the Bertini type statement of [GKKP11, Lemma 2.23] with Lemma 4.4 throughout the proof. In particular, the use of [GKKP11, Claim 17.15] needs to be modified so as to read "If $t \in T$ is a general point, then $\left(X_{t}, \Sigma_{t}\right)$ is a Du Bois pair".

The rest of the proof goes through without any change. 


\section{Preparation for the proof of Theorem (1.43)}

5.A. Split ruled surfaces. We recall some basic facts about surfaces that arise as the projectivization of a split rank two vector bundle over a curve. Let us start by fixing notation.

Notation 5.1. Let $C$ be a smooth projective curve, and let $A$ be a divisor on $C$. Form the ruled surface

$$
\pi: S=\mathbb{P}_{C}\left(\mathscr{O}_{C} \oplus \mathscr{O}_{C}(-A)\right) \rightarrow C,
$$

and let $\mathscr{O}_{S}(1)$ denote the associated relatively ample line bundle. Let $E \subset S$ be the section of $\pi$ corresponding to the surjection $\mathscr{O}_{C} \oplus \mathscr{O}_{C}(-A) \rightarrow \mathscr{O}_{C}(-A)$, and let $E_{\infty} \subset S$ be the section corresponding to the projection onto the first summand $\mathscr{O}_{C} \oplus \mathscr{O}_{C}(-A) \rightarrow \mathscr{O}_{C}$.

As $\pi$ induces an isomorphism from $E$ and from $E_{\infty}$ onto $C$, we will identify divisors on $E$, on $E_{\infty}$, and on $C$ with their images and pre-images via $\pi$. Notice that $E$ is contained in the linear system $\left|\mathscr{O}_{S}(1)\right|$, and that $\left.\mathscr{O}_{S}(E)\right|_{E} \simeq \mathscr{O}_{E}(-A)$.

Proposition 5.2 (Divisors on a ruled surface). Let $M$ be a divisor on $C$. For any $n \geq 1$, the projection formula yields an isomorphism

$$
\varphi: \bigoplus_{k=0}^{n} H^{0}\left(C, \mathscr{O}_{C}(M-k A)\right) \rightarrow H^{0}\left(S, \mathscr{O}_{S}\left(\pi^{*} M+n E\right)\right) .
$$

For some integer $k, 0 \leq k \leq n$, let $0 \neq s \in H^{0}\left(C, \mathscr{O}_{C}(M-k A)\right)$ be a nonzero section, with divisor D. Then the divisor of $\varphi(s) \in \bar{H}^{0}\left(S, \mathscr{O}_{S}\left(\pi^{*} M+n E\right)\right)$ is

$$
\pi^{*} D+(n-k) E+k E_{\infty} .
$$

Proof. We prove this proposition in three steps.

Step 1: $M=0, n=1$. In this case, the claim follows immediately from Lemma 5.3 below.

Step 2: $M=0, n$ arbitrary. Since we have

$$
\pi_{*} \mathscr{O}_{S}(n)=\operatorname{Sym}^{n}\left(\mathscr{O}_{C} \oplus \mathscr{O}_{C}(-A)\right)=\bigoplus_{k=0}^{n} \mathscr{O}_{C}(-k A),
$$

this case follows from Step 1.

Step 3: $M$ and $n$ arbitrary. The isomorphism in the projection formula is given by

$$
\begin{gathered}
\mathscr{O}_{C}(M) \otimes \pi_{*} \mathscr{O}_{S}(n) \longrightarrow \pi_{*}\left(\pi^{*} \mathscr{O}_{C}(M) \otimes \mathscr{O}_{S}(n)\right) \\
s \otimes t \longmapsto \pi^{*} s \otimes t .
\end{gathered}
$$

So this general case follows from Step 2.

Lemma 5.3 (Hyperplanes in projective space). Let $V$ be a finite-dimensional complex vector space, and let $\mathbb{P}(V)$ be the space of one-dimensional quotients of $V$. We have a canonical isomorphism

$$
\varphi: V \rightarrow H^{0}\left(\mathbb{P}(V), \mathscr{O}_{\mathbb{P}(V)}(1)\right)
$$

For any $0 \neq v \in V$, the divisor of the section $\varphi(v)$ consists of the (reduced) hyperplane

$$
\{p: V \rightarrow L \mid p(v)=0\} \subset \mathbb{P}(V) \text {. }
$$

Proof. True by definition.

5.B. Non-free linear systems. We will need a criterion for the general member of a linear system to be smooth, even though that linear system has basepoints. First we need to define a notation:

Notation 5.4. Let $X_{1}, X_{2}$ be two normal varieties and $D_{1}, D_{2}$ divisors on $X_{1}$ and $X_{2}$ respectively. We will use the notation $\left(D_{1}, D_{2}\right)$ to denote the "exterior tensor product" divisor $\operatorname{pr}_{1}^{*} D_{1}+\operatorname{pr}_{2}^{*} D_{2}$ on $X_{1} \times X_{2}$, where $\mathrm{pr}_{1}, \mathrm{pr}_{2}$ are the natural projections to $X_{1}$ and $X_{2}$.

Proposition 5.5 (Linear systems on a product). Let $S$ be a smooth projective surface, and $B$ a smooth projective curve. Let $\left|D_{1}\right|,\left|D_{2}\right|$ be linear systems on $S$ and on $B$, respectively. Assume that the schemetheoretic base locus of $\left|D_{1}\right|$ consists of a single reduced closed point $q \in S$, while $\left|D_{2}\right|$ is basepoint-free. Then a general element $T \in\left|\left(D_{1}, D_{2}\right)\right|, T \subset S \times B$, is smooth. 
The proof relies on the following easy lemma.

Lemma 5.6 (Image of a smooth divisor). Let $C \subset X$ be a smooth curve in a smooth threefold. Let $\pi: \widetilde{X} \rightarrow X$ be the blowup of $X$ along $C$, and $F_{p}=\pi^{-1}(p)$ the fibre over $p \in C \subset X$. If $D \subset \widetilde{X}$ is a smooth divisor such that $D \cdot F_{p}=1$ and $F_{p} \not \subset D$ for all $p \in C$, then $D_{X}:=\pi(D) \subset X$ is also smooth.

Proof. The assumption on the intersection between $D$ and $F_{p}$ implies that $\left.\pi\right|_{D}: D \rightarrow D_{X}$ is an isomorphism.

Proof of Proposition 5.5. Let $\pi^{\prime}: \widetilde{S} \rightarrow S$ be the blowup of $S$ at $q$, with exceptional divisor $E$, and set $\pi=\pi^{\prime} \times$ id: $\widetilde{S} \times B \rightarrow S \times B$. Then we have a decomposition into movable and fixed part

$$
\left|\pi^{*}\left(D_{1}, D_{2}\right)\right|=\left|M_{0}\right|+(E \times B),
$$

where $\left|M_{0}\right|$ is basepoint-free and one may choose a general element $M \in\left|M_{0}\right|$, such that $T=\pi(M)$. We will apply Lemma 5.6 to conclude that $T$ is smooth.

By Bertini's theorem [Har77, Ch. III, Cor. 10.9], $M$ is smooth. Furthermore, $M \cdot F_{p}=-(E \times B) \cdot F_{p}=$ $-E^{2}=1$. Finally, consider the divisor $\left.M\right|_{E \times B}$. By Bertini again, it is also smooth. Hence, if it contained a fibre $F_{p}$, it would have to be a finite union of such fibres and then its image under $\pi$ would be finite. But we clearly have

$$
\pi\left(\left.M\right|_{E \times B}\right)=\{q\} \times B
$$

leading to a contradiction. This shows that the assumptions of Lemma 5.6 are satisfied and hence the proof of Proposition 5.5 is complete.

5.C. Further ancillary results. We need two more lemmas, one of them about connectedness properties of big and nef divisors and the other one about certain divisors on curves of genus 2 .

Lemma 5.7 (Connectedness of big and nef divisors). Let $X$ be a smooth projective variety of dimension $\geq 2$ and $D \subset X$ the support of an effective big and nef divisor. Then $D$ is connected.

Proof. Let $D^{\prime}$ be an effective big and nef divisor with supp $D^{\prime}=D$. By Kawamata-Viehweg vanishing, $H^{1}\left(X, \mathscr{O}_{X}\left(-D^{\prime}\right)\right)=0$. Hence from the ideal sheaf sequence of $D^{\prime} \subset X$ we obtain a surjection $H^{0}\left(X, \mathscr{O}_{X}\right) \rightarrow H^{0}\left(D, \mathscr{O}_{D^{\prime}}\right)$, which implies that $H^{0}\left(D, \mathscr{O}_{D^{\prime}}\right)=\mathbb{C}$, and therefore $D$ must be connected.

Lemma 5.8 (Theta characteristics). Let $B$ be a smooth projective curve of genus $g=2$. Then there exists on $B$ a divisor $\Theta$ of degree 1 with the following properties:

(5.8.1) $2 \Theta \sim K_{B}$ is a canonical divisor,

(5.8.2) $h^{0}(B, \Theta)=h^{1}(B, \Theta)=0$,

(5.8.3) $h^{0}(B, n \Theta) \neq 0$ and $h^{1}(B, n \Theta)=0$ for $n \geq 3$, and

(5.8.4) the linear system $|n \Theta|$ is basepoint-free for $n \geq 3$.

Proof. The canonical linear system $\left|K_{B}\right|$ defines a two-to-one cover $B \rightarrow \mathbb{P}^{1}$, which, by the Hurwitz formula, is ramified at exactly 6 points $R_{1}, \ldots, R_{6}$. The $R_{i}$ are the only points with the property that $2 R_{i} \sim K_{B}$. On the other hand, we have, up to linear equivalence, $2^{2 g}=16$ divisors $\Theta$ satisfying $2 \Theta \sim K_{B}$. So there exist, up to linear equivalence, 10 divisors $\Theta$ such that $h^{0}(B, \Theta)=0$ and $2 \Theta \sim K_{B}$, and we choose one of them. This implies (5.811).

By choice $h^{0}(B, \Theta)=0$, so (5.82) follows from Riemann-Roch on $B$.

By Serre duality and (5.81) it follows that $h^{1}(B, n \Theta)=h^{0}(B,(2-n) \Theta)=0$ for $n \geq 3$, and then by Riemann-Roch

$$
h^{0}(B, n \Theta)=n-1 \text {, }
$$

so (5.83) follows.

Again, by Serre duality and (5.81) it follows that for any $P \in B, h^{1}(B, n \Theta-P)=h^{0}(B,(2-n) \Theta+P)$. This is clearly 0 for $n \geq 4$, but also for $n=3$ since $\Theta \nsim P$ by choice. Then, again, by Riemann-Roch

$$
h^{0}(B, n \Theta-P)=n-2,
$$

so combining (5.8.1) and (5.8.2) we have that

$$
h^{0}(B, n \Theta-P)<h^{0}(B, n \Theta)
$$


for any point $P \in B$ and $n \geq 3$. This proves (5.844).

\section{Proof of Theorem (1.4.3)}

Theorem (1.413) follows from the following more precise result.

Theorem 6.1 (Non-Du Bois potentially Du Bois variety with $\mathbb{Q}$-Cartier $K_{X}$ ). There is a 3-dimensional $D u$ Bois pair $(X, \Sigma)$ such that $X$ is normal and has an isolated singularity such that $4 K_{X}$ is Cartier and $\Sigma \subset X$ is a Weil divisor, but $X$ is not $D u$ Bois.

The construction of $X$ can be outlined as follows. First we consider a ruled surface $S$ and a section $E \subset S$ with suitable cohomological properties according to Theorem 2.5 From $S$, we obtain a similar example $F \subset T$, where additionally $T$ is of general type. The pair $(X, \Sigma)$ is then defined to be a cone over $(T, F)$.

Proposition 6.2 (Ruled surface example). There is a smooth projective surface $S$, a smooth curve $E \subset S$, and an ample divisor $L$ on $S$, such that:

(6.2.1) For all $i, n \geq 1$, it holds that $h^{i}(S, 4 n L-E)=0$.

(6.2.2) We have $h^{1}(S, 4 L) \neq 0$.

(6.2.3) The divisor $5 L-K_{S}$ is big and nef. The scheme-theoretic base locus of the linear system $\left|5 L-K_{S}\right|$ consists of a single reduced point $b$, which does not lie on $E$.

(6.2.4) For $n \geq 2$, we have $h^{2}\left(S, K_{S}+(4 n-5) L-E\right)=0$.

Proposition 6.3 (Canonically polarized example). There is a smooth projective surface $T$, a smooth (not necessarily irreducible) curve $F \subset T$, and an ample divisor $M$ on $T$, such that the following hold.

(6.3.1) For all $i, n \geq 1$, it holds that $h^{i}(T, n M-F)=0$.

(6.3.2) We have $h^{1}(T, M) \neq 0$.

(6.3.3) The surface $T$ is canonically polarized. More precisely, $4 K_{T} \sim 5 M$.

6.A. Proof of Proposition 6.2. Since this proof is somewhat lengthy, it is divided into 7 steps.

Step 1: A hyperelliptic curve. Let $C$ be a hyperelliptic curve of genus $g=7$. By [Har77, Ch. IV, Prop. 5.3], there exists on $C$ a unique $g_{2}^{1}$, that is, a linear system of dimension 1 and degree 2. The linear system $g_{2}^{1}$ defines a two-to-one cover $f: C \rightarrow \mathbb{P}^{1}$, ramified at exactly 16 points $R_{1}, \ldots, R_{16}$.

Let $H \sim 2 g_{2}^{1}$, and let $A=R_{1}$ be one of the ramification points of $f$. Note that $2 A \sim g_{2}^{1}$, and that

$$
4(H-A) \sim 6 g_{2}^{1} \sim K_{C}
$$

is a canonical divisor on $C$ by [Har77, Ch. IV, Prop. 5.3].

We need to calculate $f_{*} \mathscr{O}_{C}$. Since a torsion-free sheaf on a smooth curve is locally free, and since the injection $\mathscr{O}_{\mathbb{P}^{1}} \hookrightarrow f_{*} \mathscr{O}_{C}$ is split by the trace map, we must have $f_{*} \mathscr{O}_{C}=\mathscr{O}_{\mathbb{P}^{1}} \oplus \mathscr{O}_{\mathbb{P}^{1}}(n)$ for some integer $n$. To calculate $n$, observe that

$$
H^{1}\left(C, f^{*} \mathscr{O}_{\mathbb{P}^{1}}(6)\right)=H^{1}\left(\mathbb{P}^{1}, f_{*} f^{*} \mathscr{O}_{\mathbb{P}^{1}}(6)\right)=H^{1}\left(\mathbb{P}^{1}, \mathscr{O}_{\mathbb{P}^{1}}(6) \oplus \mathscr{O}_{\mathbb{P}^{1}}(n+6)\right)
$$

by the Leray spectral sequence and the projection formula. Using (6.4), we see that the left-hand side is one-dimensional. So $n+6=-2$, and

$$
f_{*} \mathscr{O}_{C}=\mathscr{O}_{\mathbb{P}^{1}} \oplus \mathscr{O}_{\mathbb{P}^{1}}(-8) .
$$

Step 2: Construction and properties of $S$. Let $\pi: S=\mathbb{P}_{C}\left(\mathscr{O}_{C} \oplus \mathscr{O}_{C}(-A)\right) \rightarrow C$ and let $\mathscr{O}_{S}(1)$ denote the associated relatively ample line bundle. Take $E \subset S$ to be the section of $\pi$ corresponding to the surjection $\mathscr{O}_{C} \oplus \mathscr{O}_{C}(-A) \rightarrow \mathscr{O}_{C}(-A)$, and take $E_{\infty} \subset S$ to be the section corresponding to the projection onto the first summand $\mathscr{O}_{C} \oplus \mathscr{O}_{C}(-A) \rightarrow \mathscr{O}_{C}$. We will identify divisors on $E$, on $E_{\infty}$, and on $C$. Notice that $E$ is contained in the linear system $\left|\mathscr{O}_{S}(1)\right|$, that $\pi_{*} \mathscr{O}_{S}(E) \simeq \mathscr{O}_{C} \oplus \mathscr{O}_{C}(-A)$, and that $\left.\mathscr{O}_{S}(E)\right|_{E}=\mathscr{O}_{E}(-A)$. See also Notation 5.1 
Step 3: Definition and ampleness of $L$. Let $L=\pi^{*} H+E$. We use the Nakai-Moishezon criterion to show that $L$ is ample. Let $D \subset S$ be an irreducible curve. If $D=E$ or $D$ is a fibre of $\pi$, then $L \cdot D=3$ or 1 , respectively, hence we may assume that neither is the case. Then $\pi^{*} H \cdot D>0$ and $E \cdot D \geq 0$, so $L \cdot D>0$ again. Finally observe that $L^{2}=7>0$. So $L$ is ample.

Step 4: Proof of (6.211). Let $i, n \geq 1$. Since $4 n L-E=\pi^{*}(4 n H)+(4 n-1) E$, this divisor intersects the fibres of $\pi$ non-negatively, so $R^{1} \pi_{*} \mathscr{O}_{S}(4 n L-E)=0$ and we obtain

$$
H^{i}\left(S, \mathscr{O}_{S}(4 n L-E)\right) \simeq H^{i}\left(C, \pi_{*} \mathscr{O}_{S}(4 n L-E)\right) .
$$

This already proves (6.21) if $i \geq 2$, hence we may assume that $i=1$. Using the identification $\mathscr{O}_{S}(E) \simeq$ $\mathscr{O}_{S}(1)$ we have

$$
\pi_{*} \mathscr{O}_{S}(4 n L-E)=\mathscr{O}_{C}(4 n H) \otimes \pi_{*} \mathscr{O}_{S}(4 n-1)=\bigoplus_{k=0}^{4 n-1} \mathscr{O}_{C}(4 n H-k A) .
$$

For $n, k$ in the relevant range, the degree of $4 n H-k A$ is at least $16 n-(4 n-1)=12 n+1 \geq 13>$ $\operatorname{deg} K_{C}=12$. So we have $h^{1}(C, 4 n H-k A)=0$ by Serre duality. This proves (6.211).

Step 5: Proof of (6.22). Consider the short exact sequence

$$
0 \rightarrow \mathscr{O}_{S}(4 L-E) \rightarrow \mathscr{O}_{S}(4 L) \rightarrow \mathscr{O}_{E}\left(\left.4 L\right|_{E}\right) \rightarrow 0
$$

and its associated long exact sequence. In view of (6.211), we obtain an isomorphism

$$
H^{1}\left(S, \mathscr{O}_{S}(4 L)\right) \simeq H^{1}\left(E, \mathscr{O}_{E}\left(\left.4 L\right|_{E}\right)\right) .
$$

On the other hand $\left.4 L\right|_{E} \sim 4(H-A) \sim K_{E}$ by (6.4). Since $h^{1}\left(E, K_{E}\right)=1$, (6.22) follows.

Step 6: Proof of (6.213). We have

$$
\begin{aligned}
5 L-K_{S} & =\pi^{*}(5 H)+5 E-\left(-2 E+\pi^{*}\left(K_{C}-A\right)\right) \\
& =\pi^{*}\left(5 H+A-K_{C}\right)+7 E \\
& \sim \pi^{*}\left(4 g_{2}^{1}+R_{1}\right)+7 E .
\end{aligned}
$$

By the projection formula, we obtain an isomorphism

$$
H^{0}\left(S, \mathscr{O}_{S}\left(5 L-K_{S}\right)\right)=\bigoplus_{k=0}^{7} H^{0}\left(C, \mathscr{O}_{C}\left(4 g_{2}^{1}+(1-k) R_{1}\right)\right)
$$

In order to prove the claim about the base locus, we will repeatedly apply Proposition 5.2 to produce sufficiently many divisors in $\left|5 L-K_{S}\right|$.

First, since $\left|4 g_{2}^{1}+R_{1}\right|$ has no basepoint outside $R_{1}$, taking $k=0$ we see that the base locus of $\left|5 L-K_{S}\right|$ is contained in $\pi^{-1}\left(R_{1}\right) \cup E$. On the other hand, since $\left|4 g_{2}^{1}-6 R_{1}\right|=\left|g_{2}^{1}\right|$ is basepoint-free, taking $k=7$ shows that the base locus in question is contained in $E_{\infty}$. Hence the only basepoint of $\left|5 L-K_{S}\right|$ can be at the intersection $\pi^{-1}\left(R_{1}\right) \cap E_{\infty}=\{b\}$. In particular, $5 L-K_{S}$ is nef. Calculating that $\left(5 L-K_{S}\right)^{2}=77>0$, shows that $5 L-K_{S}$ is also big.

In order to see that Bs $\left|5 L-K_{S}\right|$ is reduced, we will exhibit two members of $\left|5 L-K_{S}\right|$ smooth at $b$, with different tangent directions. To this end, note that $\left|4 g_{2}^{1}\right|$ is basepoint-free, so taking $k=1$ gives us a member of $\left|5 L-K_{S}\right|$ which is smooth at $b$, with tangent space equal to $T_{b} E_{\infty} \subset T_{b} S$. But we have already seen that $k=0$ gives a member of $\left|5 L-K_{S}\right|$ smooth at $b$, with tangent space equal to $T_{b}\left(\pi^{-1}\left(R_{1}\right)\right) \subset T_{b} S$.

It remains to show that $b$ really is a basepoint of $\left|5 L-K_{S}\right|$. Because $\left.\left(5 L-K_{S}\right)\right|_{E_{\infty}} \sim 4 g_{2}^{1}+R_{1}$, it is enough to show that $R_{1}$ is a basepoint of $\left|4 g_{2}^{1}+R_{1}\right|$. Note that $h^{0}\left(C, 4 g_{2}^{1}\right)=5$ and $h^{0}\left(C, 5 g_{2}^{1}\right)=6$ by the projection formula and (6.5). Since $\left|5 g_{2}^{1}\right|$ is basepoint-free, we have $h^{0}\left(C, 4 g_{2}^{1}+R_{1}\right)=h^{0}\left(C, 5 g_{2}^{1}-R_{1}\right)=5$. So $h^{0}\left(C, 4 g_{2}^{1}\right)=h^{0}\left(C, 4 g_{2}^{1}+R_{1}\right)$. This shows the claim and finishes the proof of (6.213). 
Step 7: Proof of (6.24). Let $n \geq 2$. By Serre duality,

$$
h^{2}\left(S, K_{S}+(4 n-5) L-E\right)=h^{0}(S,-(4 n-5) L+E) .
$$

The right-hand side vanishes since $-(4 n-5) L+E$ intersects a fiber of $\pi$ negatively. This proves (6.24) and also finishes the proof of Proposition 6.2

6.B. Proof of Proposition 6.3, Again, we divide the proof into 5 steps.

Step 1: Construction of $T$. Let $S, E$, and $L$ be as in Proposition 6.2 and let $B$ and $\Theta$ be as in Lemma 5.8 . Consider the product $X=S \times B$ with projections $\mathrm{pr}_{1}$ and $\mathrm{pr}_{2}$. We will utilize the notation from (5.4). Let $T \subset X$ be a general element of the linear system $\left|\left(5 L-K_{S}, 3 \Theta\right)\right|$. Let $F$ be the divisor $\left.\left(\operatorname{pr}_{1}^{*} E\right)\right|_{T}$, and let $M=\left.(4 L, 4 \Theta)\right|_{T}$. Clearly $M$ is ample.

Step 2: $T$ is smooth. We have $\mathrm{Bs}\left|5 L-K_{S}\right|=\{b\}$ scheme-theoretically, and $\mathrm{Bs}|3 \Theta|=\emptyset$. So by Proposition 5.5, $T$ is smooth. Also note that $T$ is connected by Lemma 5.7 .

Since $b \notin E$, the restricted linear system $\left|\left(5 L-K_{S}, 3 \Theta\right)\right|_{\mathrm{pr}_{1}^{-1}(E)}$ is basepoint-free. Hence $F \subset T$ is a smooth curve.

Step 3: Proof of (6.311). Consider the ideal sheaf sequence of $T \subset X$,

$$
0 \rightarrow \mathscr{O}_{X}\left(K_{S}-5 L,-3 \Theta\right) \rightarrow \mathscr{O}_{X} \rightarrow \mathscr{O}_{T} \rightarrow 0,
$$

and twist it by $(4 n L-E, 4 n \Theta)$, yielding

$$
0 \rightarrow \mathscr{O}_{X}\left(K_{S}+(4 n-5) L-E,(4 n-3) \Theta\right) \rightarrow \mathscr{O}_{X}(4 n L-E, 4 n \Theta) \rightarrow \mathscr{O}_{T}(n M-F) \rightarrow 0 .
$$

When calculating the cohomology groups of an exterior tensor product using the Künneth formula, we will always drop any summands where at least one factor vanishes simply for dimension reasons. Concerning the sheaf in the middle, we have

$$
h^{i}\left(\mathscr{O}_{X}(4 n L-E, 4 n \Theta)\right)=h^{i-1}(S, 4 n L-E) \cdot \underbrace{h^{1}(B, 4 n \Theta)}_{=0,[5.8[3]}+\underbrace{h^{i}(S, 4 n L-E)}_{=0,[6.2[1]} \cdot h^{0}(B, 4 n \Theta)
$$

for all $i \geq 1$, and for the sheaf on the left-hand side,

$$
\begin{aligned}
h^{i}\left(\mathscr { O } _ { X } \left(K_{S}+(4 n-5) L\right.\right. & -E,(4 n-3) \Theta))= \\
=h^{i-1}\left(S, K_{S}+(4 n-5) L\right. & -E) \cdot \underbrace{h^{1}(B,(4 n-3) \Theta)}_{=0, \sqrt{5.8[2 \sqrt{3})}}+ \\
& +\underbrace{h^{i}\left(S, K_{S}+(4 n-5) L-E\right)}_{=0 \text { if } n \geq 2, \sqrt{6.244}} \cdot \underbrace{h^{0}(B,(4 n-3) \Theta)}_{=0 \text { if } n=1, \sqrt{5.812}}
\end{aligned}
$$

for $i \geq 2$. So taking cohomology of (6.6) proves (6.31).

Step 4: Proof of (6.32). Twist the ideal sheaf sequence of $T \subset X$ by $(4 L, 4 \Theta)$, which gives

$$
0 \rightarrow \mathscr{O}_{X}\left(K_{S}-L, \Theta\right) \rightarrow \mathscr{O}_{X}(4 L, 4 \Theta) \rightarrow \mathscr{O}_{T}(M) \rightarrow 0 .
$$

We have

$$
h^{1}\left(\mathscr{O}_{X}\left(K_{S}-L, \Theta\right)\right)=h^{0}\left(S, K_{S}-L\right) \cdot \underbrace{h^{1}(B, \Theta)}_{=0, \sqrt{5.812)}}+h^{1}\left(S, K_{S}-L\right) \cdot \underbrace{h^{0}(B, \Theta)}_{=0, \sqrt{5.82}} .
$$

Furthermore,

$$
h^{1}\left(\mathscr{O}_{X}(4 L, 4 \Theta)\right) \geq \underbrace{h^{1}(S, 4 L)}_{\neq 0, \sqrt{6.222]}} \cdot \underbrace{h^{0}(B, 4 \Theta)}_{\neq 0, \underline{5.813}},
$$

so taking cohomology of (6.7) gives $h^{1}(T, M) \neq 0$, proving (6.3)2 . 
Step 5: Proof of (6.313). Since $3 \Theta=5 \Theta-K_{B}$, by the adjunction formula we have

$$
K_{T}=\left.\left(K_{X}+T\right)\right|_{T}=\left.(5 L, 5 \Theta)\right|_{T} .
$$

Claim (6.313) follows immediately. This finishes the proof of Proposition 6.3.

6.C. Proof of Theorem 6.1. Let $T, F$, and $M$ be as in Proposition 6.3. By Theorem 2.5. $(X, \Sigma)=$ $C_{a}\left(T, F, \mathscr{O}_{T}(M)\right)$ is a Du Bois pair, but $(X, \emptyset)=C_{a}\left(T, \emptyset, \mathscr{O}_{T}(M)\right)$ is not. This means that $X$ is not Du Bois. By [Kol13, Prop. 3.14], $4 K_{X}$ is a Cartier divisor and this is the smallest multiple of $K_{X}$ which is Cartier. It is clear by construction that $X$ is normal, of dimension three, and has an isolated singularity.

\section{Proof of Corollary 1.5}

Let $X$ be a variety satisfying the assumption of the Lipman-Zariski conjecture. By [Lip65, Thm. 3], $X$ is normal. Corollary 1.5 now follows immediately from Theorem 4.1 and [GK14, Thm. 1.2].

Alternatively, we may also argue as follows: If $X$ is potentially Du Bois and satisfies the assumption of the Lipman-Zariski conjecture, then $X$ is $\log$ canonical by Theorem 1.42, By [Dru14, Thm. 1.1], $X$ is smooth.

\section{REFERENCES}

[Car85] J. A. CARlson: Polyhedral resolutions of algebraic varieties, Trans. Amer. Math. Soc. 292 (1985), no. 2, 595-612. MR808740 (87i:14008)

[Dru14] S. Druel: The Zariski-Lipman conjecture for log canonical spaces, Bull. London Math. Soc. 46 (2014), no. 4, 827-835.

[DB81] P. Du Bois: Complexe de de Rham filtré d'une variété singulière, Bull. Soc. Math. France 109 (1981), no. 1, 41-81.

[Fuj13] O. FujINO: Injectivity theorems, 2013. arXiv:1303.2404 [math.AG]

[FFS13] O. Fujino, T. Fujisawa, And M. SAito: Some remarks on the semi-positivity theorems, 2013. arXiv:1302.6180 [math.AG]

[GK14] P. GRAF And S. J. Kovács: An optimal extension theorem for 1-forms and the Lipman-Zariski Conjecture, Documenta Math. 19 (2014), 815-830.

[GKK10] D. Greb, S. Kebekus, And S. J. KovÁcs: Extension theorems for differential forms and Bogomolov-Sommese vanishing on log canonical varieties, Compositio Math. 146 (2010), 193-219.

[GKKP11] D. Greb, S. Kebekus, S. J. Kovács, And T. Peternell: Differential forms on log canonical spaces, Publications Mathématiques de L'IHÉS 114 (2011), 1-83.

[GNPP88] F. Gulllén, V. Navarro Aznar, P. Pascual Gainza, and F. Puerta: Hyperrésolutions cubiques et descente cohomologique, Lecture Notes in Mathematics, vol. 1335, Springer-Verlag, Berlin, 1988, Papers from the Seminar on Hodge-Deligne Theory held in Barcelona, 1982. MR972983 (90a:14024)

[HX13] C. D. Hacon And C. XU: Existence of log canonical closures, Invent. Math. 192 (2013), no. 1, $161-195$.

[Har77] R. Hartshorne: Algebraic geometry, Graduate Texts in Mathematics, vol. 52, Springer-Verlag, New York, 1977.

[Kol13] J. Kollár: Singularities of the minimal model program, Cambridge Tracts in Mathematics, vol. 200, Cambridge University Press, Cambridge, 2013, with the collaboration of SÁndor J Kovács.

[KK10] J. Kollár and S. J. KovÁcS: Log canonical singularities are Du Bois, J. Amer. Math. Soc. 23 (2010), $791-813$.

[KM98] J. Kollár and S. Mori: Birational geometry of algebraic varieties, Cambridge Tracts in Mathematics, vol. 134, Cambridge University Press, Cambridge, 1998.

[Kov99] S. J. Kovács: Rational, log canonical, Du Bois singularities: On the conjectures of Kollár and Steenbrink, Compositio Math. 118 (1999), 123-133.

[Kov11] S. J. KovÁcs: Du Bois pairs and vanishing theorems, Kyoto J. Math. 51 (2011), no. 1, 47-69. 2784747 (2012d:14028)

[Kov12] S. J. KovÁCs: The intuitive definition of Du Bois singularities, Geometry and arithmetic, EMS Ser. Congr. Rep., Eur. Math. Soc., Zürich, 2012, pp. 257-266. 2987664

[Kov13a] S. J. KovÁcs: Singularities of stable varieties, Handbook of Moduli, Volume II, Advanced Lectures in Mathematics, vol. 25, International Press, Somerville, MA, 2013, pp. 159-203.

[Kov13b] S. J. KovÁcs: Steenbrink vanishing extended, Proceedings of 12th ALGA meeting dedicated to Aron Simis and Steven Kleiman, Bulletin of the Brazilian Mathematical Society, 2013, to appear.

[KS11] S. J. KovÁCS AND K. E. SCHWEDE: Hodge theory meets the minimal model program: a survey of log canonical and Du Bois singularities, Topology of stratified spaces, Math. Sci. Res. Inst. Publ., vol. 58, Cambridge Univ. Press, Cambridge, 2011, pp. 51-94. 2796408 (2012k:14003)

[KS13] S. J. KovÁCs AND K. E. Schwede: Inversion of adjunction for rational and Du Bois pairs, arXiv:1311.7193 [math.AG], November 2013. 
[KS12] S. J. Kovács And K. E. Schwede: Du Bois singularities deform, arXiv:1107.2349v3 [math.AG] version 3, July 2012.

[KSS10] S. J. KovÁcs, K. E. Schwede, And K. E. Smith: The canonical sheaf of Du Bois singularities, Adv. Math. 224 (2010), no. 4, 1618-1640. MR2646306 (2011m:14062)

[Lip65] J. Lipman: Free Derivation Modules on Algebraic Varieties, Amer. J. Math. 87 (1965), no. 4, 874-898.

[Lun12] V. A. Lunts: Categorical resolutions, poset schemes, and Du Bois singularities, Int. Math. Res. Not. IMRN (2012), no. 19, 4372-4420. 2981713

[Ma13] L. MA: F-injectivity and Buchsbaum singularities, arXiv:1308.0149 [math.AC], August 2013.

[Pat13] Zs. PATAKFAlvi: Semi-negativity of Hodge bundles associated to Du Bois families, arXiv:1307.5555 [math.AG], July 2013.

[PS08] C. A. M. Peters And J. H. M. Steenbrink: Mixed Hodge structures, Ergebnisse der Mathematik und ihrer Grenzgebiete. 3. Folge. A Series of Modern Surveys in Mathematics [Results in Mathematics and Related Areas. 3rd Series. A Series of Modern Surveys in Mathematics], vol. 52, Springer-Verlag, Berlin, 2008. MR2393625

[Sai00] M. SAIto: Mixed Hodge complexes on algebraic varieties, Math. Ann. 316 (2000), no. 2, 283-331. MR1741272 (2002h:14012)

[Sch12] G. Schumacher: Positivity of relative canonical bundles and applications, Invent. Math. 190 (2012), no. 1, $1-56$.

[Sch09] K. Schwede: F-injective singularities are Du Bois, Amer. J. Math. 131 (2009), no. 2, 445-473. MR2503989 (2010d:14016)

[Ste83] J. H. M. Steenbrink: Mixed Hodge structures associated with isolated singularities, Singularities, Part 2 (Arcata, Calif., 1981), Proc. Sympos. Pure Math., vol. 40, Amer. Math. Soc., Providence, RI, 1983, pp. 513536. MR713277 (85d:32044)

[Ste85] J. H. M. Steenbrink: Vanishing theorems on singular spaces, Astérisque 130 (1985), 330-341.

PG: Lehrstuhl für Mathematik I, Universität Bayreuth, 95440 Bayreuth, Germany

E-mail address: patrick.graf@uni-bayreuth.de

SJK: University of Washington, Department of Mathematics, Box 354350, Seattle, WA 98195-4350, USA

E-mail address: skovacs@uw.edu 